Interactive comment on "Importance of high resolution nitrogen deposition data for biogeochemical modeling in the western Baltic Sea and the contribution of the shipping sector" by Daniel Neumann et al.

Daniel Neumann et al.

daniel.neumann@io-warnemuende.de

Received and published: 30 November 2018

Response to the comments of Reviewer \#2

We thank the reviewer for the constructive comments on the manuscript. 
The manuscript (hereafter referred to as MS) attempts to answer two relevant ocean research questions. What resolution of atmospheric nitrogen deposition is necessary to properly evaluate its' impact and how high is the contribution from shipping? The main problem with the manuscript is, as the authors seem to be aware, that the method may not allow them to fully answer the first question.

The evaluation of different spatial resolution of the nitrogen deposition is based on model results from two years, one year spin up and one year to evaluate. A typical residence time for the evaluated region is not given, but the residence time for the Baltic Sea (which is given in the MS) is stated to be significantly higher than two years. It seems reasonable to assume that a one year spin-up is too short for the biogeochemical system to reach a steady state ready to be evaluated.

However, the manuscript reads in a clear, concise, and well-structured way, although a bit repetitious. The scientific approach is transparent and the methods and results are discussed in an appropriate way. The problem is that the overall experiment design is flawed, which is a shame.

\section{Specific comments}

The title of the manuscript, and the abstract, represents the manuscript's content well and only some bits of information are lacking to cleary present the methods (see the technical comments below). 
One of the significant pieces of information that are missing is a statement of the quality of the physical model, HBM. This can be done by stating findings in previous publication since it is not the main focus of this MS, but it needs to be stated and the relevant publication referred to. It is not necessarily so that an operational model gives good results when used to hindcast.

> We added a second paragraph to Sect. 2.2 ("Marine Modeling") providing information on the quality of the physical mode.

Another thing missing is the lack of focus on the biogeochemical processes in the model and how they are affected by changes in the resolution of nitrogen deposition. Also, a discussion of the general nutrient dynamics of the study area should be an important part of the section "2.3 Study region". Both these additions would aid in understanding the results. Especially as the validation shows large discrepancies.

$>$ added a description of basic system dynamics to Sect. 2.3 as suggested

$>$ removed "processes" from the research question

Since the study uses a constant phosphorous deposition, and aims evaluate the effect of nitrogen deposition, I assume/hope that the area is generally nitrogen limited? However, this should be stated.

> Yes, most parts of the Baltic Sea are nitrogen limited (Feistel et al., 2008, Sect. 12.3, al., 2015). However, the areas affected by river plumes and phosphorus limitation are rather small. 
$>$ added this information to Sect. 2.3 and pointed from phosphorus-depositionparagraph in Sect. 2.2 to Sect. 2.3

$>$ According to current assumption by HELCOM (2015b, "Updated Fifth Baltic Sea Pollution Load Compilation (PLC-5.5)"), atmospheric phosphorous deposition contributes approximately $5 \%$ to the total phosphorous input into the Baltic Sea. Therefore, phosphorous deposition has a lower contribution than nitrogen deposition with respect to the total inputs. However, there is large uncertainty in phosphorous deposition estimates and a few colleagues expect the phosphorous deposition to be higher.

The validation section does not really state that the model results are good enough to answer the research question. Are they? Is there too little data? The validation section should have a clear and well-argued conclusion. The authors also leave too much important information to be available in other publications/submissions only. The reader should be given the most important info for this MS in a sentence or two and not only the statement that it is elsewhere. See more specific comments about this in the technical corrections section.

$>$ We added a summarizing paragraph to the validation section as suggested.

$>$ We added information from cited publications - i.e. Bian et al. (2017) and Karl et al. $(2018 a, b)-$ to the text as documented in our answers to the specific comments below.

The conclusions section is too long and contains too much discussion and outlook.

$>$ We renamed the conclusions to summarizing discussion, included the suggested

Finally I'm skeptical to be a given a residence time valid for the Baltic Sea. Is 
it applicable also for the shallow Belt Sea, the southern Kattegat ect. ? If so, please state it.

> The residence time cited from Radtke et al. (2012) should be considered as an upper limit because the region of interest is quite shallow - as the reviewer mentions. We modified the respective paragraphs in the Section 3.2.3 (last paragraph) and in the OSD

Conclusions.

\section{Technical corrections}

Page 2. Line 6: Change the " $\AA$ " to lower case.

$>$ corrected as suggested

Page 2, line 21: Remove "the" before "land".

$>$ removed as suggested

Page 2, line 31: Change "it" to "it's" or "it is".

$>$ changed as suggested

Page 2, line 31: "Example of a region", not "for".

$>$ corrected as suggested

(c) (i) 
Page 3, line 17: The sentence feels unfinished.

$>$ We changed "However, shipping traffic is also expected to increase in the Baltic Sea in the next decades (e.g., Matthias et al., 2016; Karl et al., in prep. same spec. issue a)." to "However, shipping traffic is also expected to increase in the Baltic Sea in the next decades and cargo vessels have a life time of approximately 25 to 30 years (e.g., Buhaug et al., 2009; Matthias et al., 2016; Karl et al., in prep., a; Smith et al., 2014; Danish EPA, 2012). Therefore, the expected reduction in overall shipping $\mathrm{NO}_{\mathrm{X}}$ emissions is rather low in the next decade (e.g., Geels et al., 2012; Jonson et al., 2015; Hammingh et al., 2012)."

Page 4, line 1: Consider removing the commas around "this is the first study". At least the second comma seems out of place.

> removed commas as suggested; we restructured this paragraph and moved this sentence to the end of the paragraph;

Page 4, line 13-14: "Based on ... is performed". The formulation is out of place. Please, consider rewriting it.

> replaced "Based on ... is performed" by "The impact of spatially refined nitrogen deposition on the biogeochemical model results was assessed by evaluating two HBMERGOM model simulations forced by these two deposition data sets. These HBMERGOM simulations are named CMAQ16mod and CMAQ04mod, respectively, corresponding to the deposition data sets."

Page 5, line 26: Please add the relevant information from the validation. I.e. are the cmaq04 simulation results sufficiently good? Are there any shortcoming the reader needs to know about?
OSD

Interactive

comment
Printer-friendly version

Discussion paper 
$>$ The nitrogen wet deposition was underestimated compared to EMEP measurements in the Baltic Sea region. However, the atmospheric $\mathrm{NO}_{x}$ were well reproduced. The reason for the underestimation of the wet deposition was not fully resolved. We extended the paragraph.

Page 6, line 3-4: And what do Bian et al (2017) say? Since the EMEP model results are used in this MS you should shortly mention the essence of the comparison. If it is of no importance, why mention it at all?

$>$ Thanks for the remark. We included a paragraph summarizing the results of Bian et al. (2017).

Page 6, line 16-17: Does HBM work well in hindcast mode? Please state and/or refer to some info about the model skill.

$>$ We appended the following sentences to the paragraph: "Sea surface temperature and salinity are generally well predicted according to recent publications (Wan et al., 2012; Bruening et al., 2014. However, Wan et al. (2012) identified a drift of salinity in depths $>75 \mathrm{~m}$ of the central Baltic Sea in a two-year hindcast indicating too strong vertical mixing. In preliminary multi-year simulations we found similar evidence. Because we evaluate a short time span and surface water concentrations only, we expect no critical interference through this issue."

Page 9, line 7: I think it would be beneficial to state in the "2.4 Model validation" section that the model validation will be done for all three runs.

> changed sentence on p.9 I.8 from "The biogeochemical model results were validated against measurements." to "The results of all three HBM-ERGOM simulations were validated against measurements." 
Page 11, line 11: Remove "the" before "land".

OSD

$>$ removed as suggested

Page 12, line 20: Replace "few" with "little" or any other word meaning "not much".

Interactive "Few" is used for "not many".

$>$ corrected as suggested

Page 13, line 3: Replace "few" with "little" or any other word meaning "not much". "Few" is used for "not many".

$>$ corrected as suggested

Page 13, line 3-4: Since the winter DIP levels seem more correct than the winter DIN levels, l'd say that the high summer DIP concentrations are caused by too little DIN, not too much DIP, or possibly that there are cyanobacteria in reality, but not in the model. Something similar is stated a few lines down, but the argument is sort of started in the wrong way. Please rewrite.

$>$ rewritten as suggested

$>$ added a sentences on this topic to the "Summarizing Discussion" (previously "Conclusions")

Printer-friendly version

Page 14, line 3: Replace "few" with "little" or any other word meaning "not much". "Few" is used for "not many". 
Page 14, line 31: It is unclear if PON includes living biomass, i.e. phyto and zooplankton, or only dead particulate organic nitrogen. Does it? I suppose living matter is bio available, but it reads strange.

Interactive comment

$>$ Yes, PON as we use it includes living biomass. We explicitely state "bioavailable" because we do not consider PON that is somehow not available for biogeochemical processes. Clarified the meaning of "bioavailable PON" and added how PON is calculated (detritus + phytoplankton + zooplankton).

Page 15, line 9-10: The sentence: "This is reasonable ... and vertical stratification" makes me think PON includes living organic particulates, otherwise it makes no sense?

> Yes. Please see reply to previous comment.

Page 17, line 13-15: I'm skeptical to be a given only a residence time valid for the Baltic Sea. Is it applicable also for the shallow Belt Sea, the southern Kattegat ect. ?

$>$ We performed additional three years of simulation and saw that atmospheric nitrogen converges to a steady state after two to three years in this region. We, now, consider the numbers of Radtke et al. (2012) as upper limit. Therefore, the two-years period evaluated in this study seems to by only just sufficient.

$>$ We split the paragraph into two and modified the text according to the new findings. 
$>$ The whole paragraph was reformulated. Originally, we wanted to express that the steady-state atmospheric nitrogen concentration is not reached yet. It was badly forOSD mulated.

Page 18, line 8: Mai=May ?

Interactive

comment

$>$ corrected as suggested

Page 19, fig 10: Figure it too small.

> increased size and included it as vector graphic (before it was a PNG)

Page 20, line 8-12: But what did those publications find? Even if the evaluation work in itself is not part of this study the result is certainly important. Please use a few sentences to state the main findings instead of using two to just say that the information is elsewhere. E.g. : "Karl et al (in prep ... issue a) found that ... and the same study also showed that..."

$>$ reformulated second half of paragraph and moved to summary (now first paragraph of summary)

Page 20, line 12-13: Repeated sentence.

$>$ removed as suggested

Page 20, line 28-29: Isn't EMEP better/less bad (Fig 6)? 
$>$ We agree that summer DIP concentrations and winter DIN are closer to the measurements in the EMEP50mod case. A sentence was added to the new Sect. 3.5 (Summarizing Discussion) "..., EMEP50mod DIN and DIP concentrations were closer to the measurements in these particular time periods."

Page 20, line 30: Why do you use a time period (April - September) when the DIN levels are likely to be depleted and thus depend to a large degree on the biogeochemical model, not so much the input? Wouldn't it be better to use winter months for DIN? If it is changes in phytoplankton growth/production you are after I suggest you show assimilation by the phytoplankton or maybe the export production or such?

$>$ We agree. Now, we show annual means to cover the nutrient-rich period in winter and the phytoplankton-rich period in summer. Unfortunately, we did not write out nutrient consumption/release per process. Hence, a detailed evaluation of net process flows is not possible without re-running the simulations.

Interactive comment on Ocean Sci. Discuss., https://doi.org/10.5194/os-2018-71, 2018. 